Department of Public Health, University of

Liverpool,

Liverpool L69 3GB

Simon Capewell professor of clinical epidemiology

Correspondence to: J Chalmers jim.chalmers@isd. csa.scot.nhs.uk practice at the University of Glasgow, is helpful: "More miles on the clock" (G Watt, personal communication, 1999).

The composition of the cohort changed markedly from 1974 to 1997, with a much greater proportion of survivors in 1997 being in the least deprived fifth than in the most deprived ( $27 \%$ and $14 \%$, respectively, of men and $23 \%$ and $17 \%$ of women).

These effects of deprivation on premature death may not be obvious in tables or histograms. ${ }^{2}$ Our cohort survival graphs, which can be constructed without complex links between records, clearly show the relation between deprivation and mortality.
We thank the General Register Office for providing the mortality and population data and David Murphy of the Information and Statistics Division for his help in the initial analyses.

Contributors: JC had the original idea, which he then developed with SC. JC did the analyses. Both authors drafted and wrote the paper.JC is the guarantor for the paper.

Competing interests: None declared.

1 Langmuir AD. William Farr: founder of modern concepts of surveillance. Int J Epidemiol 1976;5:13-8.

2 Loevinsohn BP. Data utilization and analytical skills among mid-level health programme managers in a developing country. Int J Epidemiol 1994;23:194-200.

3 Carstairs V, Morris R. Deprivation and health in Scotland. Aberdeen: Aberdeen University Press, 1991.

4 Hart CL, Smith GD, Blane D. Social mobility and 21 year mortality in a cohort of Scottish men. Soc Sci Med 1998;47:1121-30.

(Accepted 21 September 2001)

\title{
Mobility impairments and use of preventive services in women with multiple sclerosis: observational study
}

Eric Cheng, Lawrence Myers, Sheldon Wolf, Deborah Shatin, Xin-Ping Cui, George Ellison, Thomas Belin, Barbara Vickrey

Editorial by Wagner

Department of

Neurology,

University of

California,

650 Charles Young

Drive South,

Box 951736,

Los Angeles,

CA 90095-1769,

USA

Eric Cheng

health services fellow

Lawrence Myers

professor

Sheldon Wolf

physician

George Ellison

professor emeritus

Barbara Vickrey

associate professor

Center for Health

Care Policy and

Evaluation, 9000

Bren Road East,

Minnetonka

MN 55343, USA

Deborah Shatin

senior researcher

Department of

Biostatistics,

University of

California,

Los Angeles

Xin-Ping Cui

graduate student

Thomas Belin

associate professor

Correspondence to:

E M Cheng

emc@ucla.edu

BMJ 2001;323:968-9
Use of preventive health services is affected by factors such as patient demographics, clinical characteristics, type of provider, and type of healthcare system. ${ }^{1}$ Although people with multiple sclerosis may have impaired mobility, their lifespans are similar to age matched population controls. They therefore need standard preventive services to prevent early mortality. We evaluated the relation between mobility and use of preventive services in women with multiple sclerosis.

\section{Participants, methods, and results}

In 1996, we sent questionnaires to 1164 adults with multiple sclerosis who had received outpatient care in 1993 or 1994 from one of three systems of health care (two forms of managed care and fee for service insurance) in two regions of the United States. ${ }^{2}$ The overall response rate was $80 \%(930 / 1164)$. We report here survey analyses from the 713 women respondents.

We collected self reported rates of cervical smear testing, mammography, and breast examination (if over age 50), blood pressure checks, cholesterol screening, and physician assessment of health habits. We assessed these rates according to the patient's mobility level (fully ambulatory, ambulatory with help, and not ambulatory) and compared them with Healthy People 2000 recommendations. ${ }^{3}$ For each preventive service, we used logistic regression to model the relation between that service, mobility, patient demographics, comorbidity, ${ }^{2}$ system of health care, indicators for having a primary care physician and a multiple sclerosis physician, and specialty of these physicians.

The mean age of the women was 47 years; $86 \%$ were white and $40 \%$ had a four year college degree. Overall rates for cervical smear tests, breast examinations, and mammography exceeded Healthy People 2000 recommendations, but rates were highest for the ambulatory group and lowest for the non-ambulatory group ( $\mathrm{P} \leqslant 0.05$, table). Cervical smear testing was below Healthy People 2000 goals for the ambulatory with help and non-ambulatory groups. In contrast, rates for general preventive services did not differ by mobility.

In the multivariable models, ambulatory patients had 5.32 times the odds of having a cervical smear test, 3.62 times the odds of having a breast examination, and 3.24 times the odds of having mammography relative to non-ambulatory patients (all $\mathrm{P}<0.05$ ). Older age was associated with a lower rate of cervical smear tests; however, no other variables were related to receipt of women's preventive services. Except for an increased odds of assessing eating habits in the non-ambulatory group, mobility status did not affect the odds of receiving general preventive services.

\section{Comment}

Use of women's preventive health services was lower in non-ambulatory women than in fully or partially ambulatory women with multiple sclerosis. These results are the same as those in a previous population based US study of women with and without mobility impairments due to various conditions, ${ }^{4}$ even though women in our study had a single chronic condition, were younger, were more educated, and all had health insurance and a regular source of care in health systems that met broad national screening goals.

There are several possible explanations for these findings. Doctors may believe that such patients do not have an adequate life expectancy to warrant women's preventive screening. However, such attitudes would be incompatible with the high rates of blood pressure and cholesterol checks. Alternatively, patients may be reluctant to undergo screening services that are potentially uncomfortable or embarrassing. ${ }^{5}$ A third possibility is that the medical systems cannot easily accommodate patients with mobility impairments, who may require access to specialised equipment and extra time. 
Use of preventive services among women with multiple sclerosis by degree of mobility

\begin{tabular}{|c|c|c|c|c|c|c|c|c|c|}
\hline & \multirow{2}{*}{$\begin{array}{c}\text { Healthy } \\
\text { People } \\
2000 \\
\text { target } \\
\text { rates }(\%)\end{array}$} & \multicolumn{4}{|c|}{ No $(\%)$ reporting use of preventive services (unadjusted)* } & \multirow[b]{2}{*}{$\begin{array}{c}P \\
\text { value }\end{array}$} & \multirow{2}{*}{$\begin{array}{l}\text { Validation } \\
\text { (c) statistic } \\
\text { for logistic } \\
\text { regression }\end{array}$} & \multicolumn{2}{|c|}{ Adjusted odds ratios $(95 \% \mathrm{Cl}) \pi$} \\
\hline & & $\begin{array}{c}\text { Overall } \\
(\mathrm{n}=713)\end{array}$ & $\begin{array}{c}\text { Fully } \\
\text { ambulatory } \\
(\mathrm{n}=363)\end{array}$ & $\begin{array}{c}\text { Ambulatory } \\
\text { with help } \\
(\mathrm{n}=239)\end{array}$ & $\begin{array}{c}\text { Not } \\
\text { ambulatory§ } \\
(\mathrm{n}=111)\end{array}$ & & & Fully ambulatory & Ambulatory with help \\
\hline \multicolumn{10}{|l|}{ Women's preventive health services } \\
\hline $\begin{array}{l}\text { Cervical smear test in past } \\
3 \text { years }\end{array}$ & 85 & $585 / 692(85)$ & $334 / 361(93)$ & $181 / 228(79)$ & $70 / 103(68)$ & 0.001 & 0.73 & 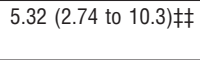 & 2.11 (1.15 to 3.85$)$ 㧊 \\
\hline Breast examination in past year ${ }^{* *}$ & 60 & $196 / 245(80)$ & $83 / 96(86)$ & $82 / 104(79)$ & $31 / 45(69)$ & 0.05 & 0.67 & 3.62 (1.39 to 9.42$)$ & 1.83 (0.75 to 4.48$)$ \\
\hline \multicolumn{10}{|l|}{ General preventive health services } \\
\hline $\begin{array}{l}\text { Blood pressure checked in past } \\
\text { year }\end{array}$ & $90+\dagger$ & $532 / 678(78)$ & $268 / 351(76)$ & $179 / 223(80)$ & $85 / 104(82)$ & 0.37 & 0.70 & $0.83(0.43$ to 1.59$)$ & $0.78(0.39$ to 1.53$)$ \\
\hline $\begin{array}{l}\text { Cholesterol checked in past } \\
5 \text { years }\end{array}$ & 75 & $494 / 626(79)$ & $258 / 326(79)$ & $164 / 205(80)$ & $72 / 95(76)$ & 0.70 & 0.72 & 1.31 (0.69 to 2.49$)$ & 1.17 (0.61 to 2.26$)$ \\
\hline \multicolumn{10}{|l|}{ In past year, a doctor asked about: } \\
\hline Eating habits & & $207 / 631$ (33) & $100 / 329(30)$ & $66 / 206(32)$ & $41 / 96(43)$ & 0.08 & 0.63 & $0.44(0.25$ to 0.76$)$ & $0.53(0.30$ to 0.93$)$ \\
\hline Exercise habits & & $327 / 639(51)$ & $172 / 336(51)$ & $111 / 209(53)$ & $44 / 94(47)$ & 0.60 & 0.61 & 0.86 (0.51 to 1.47$)$ & $1.00(0.58$ to 1.73$)$ \\
\hline
\end{tabular}

*Response rates exclude women who answered "not sure." When women who responded not sure were combined with those who responded no, the results were similar.

†Can walk at least 1 block or $90 \mathrm{~m}$ without ambulation aid.

tCan walk at least $4.5 \mathrm{~m}$ with the aid of a cane, crutch, brace, or walker.

§Unable to walk $4.5 \mathrm{~m}$ with the aid of a cane, crutch, brace, or walker.

ПCompared with not ambulatory group. Covariates include mobility, age, race, education, comorbidity, system of health care, indicators for having a primary care physician and multiple sclerosis physician, and specialty of these physicians. Interaction terms of mobility and health system, and mobility and education were included in models but were not significant.

**For respondents aged $\geqslant 50$ years.

t+Healthy People $2000 \mathrm{goal}$ is for $90 \%$ of adults to have had blood pressure checked in past 2 years.

$\ddagger \neq T$ The fully ambulatory group also had significantly higher odds of smear testing relative to the "ambulatory with help" group.

Women with impaired mobility should be considered a vulnerable population for receipt of breast examinations, mammography, and cervical smear tests. Studies are needed to identify factors causing this and to evaluate interventions to reduce the variation across mobility levels.

We thank Robert Brook for advice in designing the protocol and contributing to the interpretation of results, Martin Shapiro for help in designing the survey, and Rebecca Hanson for help in designing the survey protocol and collecting data.

Contributors: EC participated in the data analysis, data interpretation, and writing the paper. XC participated in the data analysis and data interpretation. TB, GE, LM, DS, and SW participated in designing the protocol, data collection, data interpretation, and revising the paper. BV had the original idea for this study and participated in the data analysis, data interpretation, and writing the paper. All authors contributed to writing the paper. $\mathrm{EC}$ and $\mathrm{BV}$ will act as guarantors.
Funding: This work was supported by the Veteran's Administration, the Robert Wood Johnson Foundation, the health care delivery and policy research program of the National Multiple Sclerosis Society (contract HC 0003), and the National Institute for Neurological Disorders and Stroke (K08 NS01669).

Competing interests: None declared.

1 Frame PS. Health maintenance in clinical practice: strategies and barriers. Am Fam Physician 1992;45:1192-200.

2 Vickrey BG, Shatin D, Wolf SM, Myers LW, Belin TR, Hanson RA, et al Management of multiple sclerosis across managed care and fee-forservice systems. Neurology 2000;55:1341-9.

3 United States Department of Health and Human Services. Healthy people 2000: national health promotion and disease prevention objectives. Hyattsville, MD: USDHHS, 1995

4 Iezzoni LI, McCarthy EP, Davis RB, Siebens H. Mobility impairments and use of screening and preventive services. Am J Public Health 2000;90:95561

5 Becker H, Stuifbergen A, Tinkle M. Reproductive health care experiences of women with physical disabilities: a qualitative study. Arch Phys Med Rehabil 1997;78(suppl 5):S26-33.

(Accepted 5 September 2001)

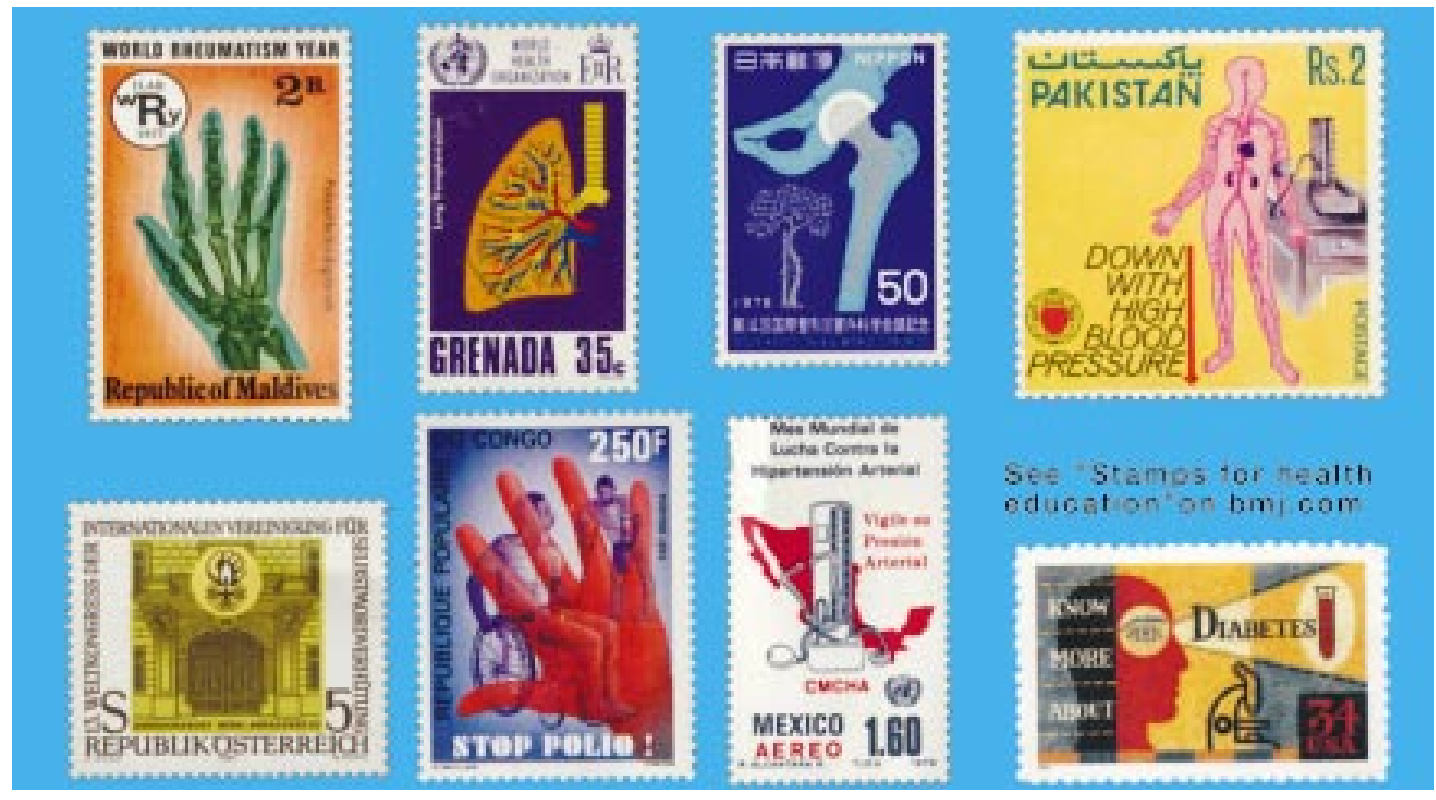

